\title{
Oncogenic Ras/squamous cell carcinoma antigen signaling pathway activation promotes invasiveness and lymph node metastases in papillary thyroid carcinoma
}

\author{
DINGYUAN LUO ${ }^{1-3^{*}}$, YING LIU ${ }^{4 *}$, SHAOJIAN LIN ${ }^{*}$, LANGPING TAN $^{1}$, ZEHU DU $^{1}$, MIAOYUN LONG $^{1}$, \\ YUE ZHU ${ }^{1}$, XINZHI PENG ${ }^{1}$, WEIXING ZONG ${ }^{5}$, GERARDO G. MACKENZIE ${ }^{3}$, \\ HONGHAO LI ${ }^{1}$ and NENGTAI OUYANG ${ }^{6}$ \\ ${ }^{1}$ Department of Thyroid Surgery, Sun Yat-Sen Memorial Hospital, Sun Yat-Sen University; \\ ${ }^{2}$ Guangdong Provincial Key Laboratory of Malignant Tumor Epigenetics and Gene Regulation, \\ Medical Research Center, Sun Yat-Sen Memorial Hospital, Sun Yat-Sen University, Guangzhou, \\ Guangdong 510120, P.R. China; ${ }^{3}$ Department of Nutrition, University of California, Davis, CA 95616, USA; \\ ${ }^{4}$ Department of Anesthesiological Recovery, Sun Yat-Sen Memorial Hospital, Sun Yat-Sen University, \\ Guangzhou, Guangdong 510120, P.R. China; ${ }^{5}$ Department of Chemical Biology, Rutgers University, Piscataway, \\ NJ 08854, USA; ${ }^{6}$ Department of Cellular and Molecular Diagnostics Center, Sun Yat-Sen Memorial Hospital, \\ Sun Yat-Sen University, Guangzhou, Guangdong 510120, P.R. China
}

Received July 30, 2018; Accepted November 8, 2018

DOI: $10.3892 /$ or.2018.6895

\begin{abstract}
Papillary thyroid carcinoma (PTC) is a type of cancer with one of the fastest increasing incidences worldwide. However, the therapeutic choices for PTC patients are limited and it is critical to further understand the molecular pathology underlying this disease. Squamous cell carcinoma antigens (SCCAs) are overexpressed in many tumors and participate in tumorigenesis. However, their roles in PTC are incompletely understood. Therefore, this study investigated the role of SCCA in PTC, evaluating its expression, its clinical implications and
\end{abstract}

Correspondence to: Dr Nengtai Ouyang, Department of Cellular and Molecular Diagnostics Center, Sun Yat-Sen Memorial Hospital, Sun Yat-Sen University, 107 Yanjiang West Road, Guangzhou, Guangdong 510120, P.R. China

E-mail: ouynt@mail.sysu.edu.cn

Dr Honghao Li, Department of Thyroid Surgery, Sun Yat-Sen Memorial Hospital, Sun Yat-Sen University, 107 Yanjiang West Road, Guangzhou, Guangdong 510120, P.R. China

E-mail: lihonghao158158@163.com

*Contributed equally

Abbreviations: SCCA, squamous cell carcinoma antigen; PTC, papillary thyroid carcinoma; LNM, lymph node metastasis; FBS, fetal bovine serum; IHC, immunohistochemistry; MOD, mean optical density; MAPK, mitogen-associated protein kinase; OS, overall survival; DFS, disease-free survival; LNRFS, lymph node recurrence-free survival; DRFS, distant recurrence-free survival

Key words: SCCA, Ras, invasion, metastasis, papillary thyroid carcinoma prognostic significance in PTC patient samples, as well as its function in vitro and in vivo, using a thyroid cancer cell line in which SCCA levels have been knocked down or overexpressed. In this study, SCCA expression levels were measured by immunohistochemistry (IHC) in non-cancerous and tumor tissues. Kaplan-Meier analyses assessed the survival in PTC patients. MTT assay, western blot analysis, invasion assay and xenograft tumor assay were used to calculate cell proliferation, migration, invasion and tumor growth. Our results showed that SCCA was overexpressed in PTC tissues and was correlated with the clinical stage of PTC. Patients with high SCCA expression had lower overall survival (OS), disease-free survival (DFS), lymph node recurrence-free survival (LNRFS), and distant recurrence-free survival (DRFS), compared to patients expressing low level of the SCCA protein. SCCA knockdown suppressed thyroid cancer cell proliferation, invasion and reduced xenograft tumor growth, whereas SCCA overexpression increased cell proliferation, invasion and xenograft tumor growth. Mechanistically, the activation of Ras increased SCCA expression, and SCCA expression was positively correlated with Ras levels in the PTC tissues. In conclusion, SCCA protein is overexpressed in PTC and may represent a predictive prognostic factor for PTC patients. Furthermore, activation of the Ras/SCCA pathway plays an important role in promoting tumor growth, invasion and metastasis in PTC.

\section{Introduction}

Papillary thyroid carcinoma (PTC) is the most common type of differentiated thyroid carcinoma, accounting for more than $80 \%$ of all thyroid malignancies, presenting as a frequent type of cancer in many parts of the world, including China (1-3). An important characteristic of PTC is that it is prone to spread 
through lymphatic ducts, resulting in recurrence, metastases, and even patient death. Although multi-kinase or tyrosine kinase inhibitors have been approved for the treatment of radioiodine-refractory PTC (4), the therapeutic choices are limited due in part to the lack of understanding of the molecular characteristics and pathophysiology of PTC $(5,6)$. Therefore, it is critical to further understand the molecular characteristics underlying PTC progression in order to identify useful diagnostic biomarkers as well as potential targets for therapy.

Squamous cell carcinoma antigens (SCCAs) are members of the serpin family of endogenous protease inhibitors. The first variant of SCCA (SCCA1) was initially found to be overexpressed in squamous cell carcinoma (SCC) of the uterine cervix (7). A previous study showed that SCCA1 and its isoform, SCCA2, were produced by two tandemly arranged genes located on chromosome 18q21 (8), being 98 and 92\% homologous at their nucleotide and amino acid levels, respectively $(9,10)$. Apart from being present in cervical SCC, SCCA has been shown to be frequently overexpressed in tumors of different histological types including breast carcinoma (11), liver (12) and lung cancer $(9,13)$, head and neck carcinoma (14), and esophageal cancer (15). In addition, circulating SCCA is currently used as a valuable predictor of node metastasis, response to treatment, and tumor recurrence $(16,17)$. The SCCA level has been shown to predict pathological grade, disease stage, recurrence, and response to both radiotherapy and chemotherapy. SCCA expression has also been associated with poorly differentiated and advanced metastatic SCCs $(18,19)$. However, the biological role of SCCA in PTC and its clinical significance have not been explored.

The Ras family of small GTPases, consisting of three highly related members, K-Ras, H-Ras and N-Ras, is a central regulator of growth, proliferation and differentiation processes in virtually every nucleated cell. Being located in the inner leaflet of the plasma membrane, Ras GTPases play an important role in the transmission of signals through interaction with multiple effectors. Activation of Ras is initiated by cell surface receptors, which can induce Ras GEFs to exchange GDP with GTP on Ras. Then Ras stimulates diverse downstream effectors, resulting in the initiation of an array of cellular signaling networks, including the RAF-1/MEK/ERK, PI3K/Akt/mTOR and Rac1/JNK pathways (20-22). Collectively, Ras mutations and pathway activation induce dramatic changes to cells including promotion of proliferation, suppression of apoptosis, increased cell invasiveness and metabolic rewiring (23).

Activating Ras mutations are found in $\sim 25 \%$ of all human tumors, but these three small GTPases are not mutated at equivalent frequencies in cancer. Eighty-five percent of Ras-driven cancers have activating mutations in K-Ras, while $\mathrm{N}$-Ras and $\mathrm{H}-\mathrm{Ras}$ are mutated in 12 and $3 \%$, respectively, of all cancers $(24,25)$. Ras mutations are common in pancreatic ductal tumors (26), colorectal tumors $(27,28)$, PTC $(29,30)$ and lung cancer (31). In PTC, RAS mutations may promote thyroid tumorigenesis through the verified Ras/Raf/MEK pathway or through its interaction with the PI3K/AKT pathway (32). In addition, Ras mutations are associated with tumor metastases in PTC $(29,33)$. Notably, in our previous study, Ras significantly increased SCCA protein levels in colon cancer cell lines, and SCCA was found to be transcriptionally upregulated by oncogenic Ras through the MAPK pathway (34).
In the present study, we examined, using PTC cells, xenograft models and PTC patient samples, the role of SCCA and Ras in PTC progression and metastasis. Our data indicate that SCCA is upregulated in human PTC tissues and is significantly correlated with tumor stage. In mice, SCCA overexpression accelerated thyroid cancer xenograft growth, while SCCA suppression reduced xenograft growth. Furthermore, in in vitro studies, Ras upregulatedd SCCA expression and promote cell proliferation, migration and invasion in PTC cells. Notably, both Ras and SCCA protein are overexpressed and are significantly associated with tumor stage in PTC. Moreover, Cox correlation analysis showed that SCCA may be used as an independent prognostic factor for PTC patients. In conclusion, these results suggest that SCCA protein upregulated by Ras accelerates the invasion and metastasis in PTC, and that SCCA could be utilized as a potential target for PTC treatment.

\section{Materials and methods}

Human tissue samples and follow-up. Human tissue samples were obtained through surgical resection at Sun Yat-Sen Memorial Hospital of Sun Yat-Sen University from June, 2006 to July, 2009. All tissue specimens were collected, frozen and stored in liquid nitrogen until assayed. All tissue samples were obtained with informed consent, and all procedures were performed in accordance with the Internal Review and the Ethics Board of the Sun Yat-Sen Memorial Hospital of Sun Yat-Sen University. All clinical information was obtained from patients during follow-up. Moreover, overall survival (OS) was calculated as the time from the date of diagnosis to the date of death or the date of the last follow-up (if death did not occur). Disease-free survival (DFS) was calculated as the time from the date of surgery to the date of the first recurrence or metastasis after surgery (in patients with recurrence or metastasis) or to the date of the last follow-up (in patients without recurrence and metastasis). Lymph node recurrence-free survival (LNRFS) was defined as the time from the date of surgery to the date of lymph node relapse, and distant recurrence-free survival (DRFS) was defined as the time from the date of surgery to the date of distant recurrence. Clinical information of the samples is shown in detail in Table I.

Immunohistochemistry (IHC). SCCA protein expression was analyzed by immunohistochemistry (IHC) on paraffinembedded PTC tissue samples as previously described in our study (35). Briefly, the specimens were cut into $5-\mu \mathrm{m}$ sections and baked at $65^{\circ} \mathrm{C}$ for $30 \mathrm{~min}$. The sections were deparaffinized and antigenic retrieval was carried out. The sections were treated with $3 \%$ hydrogen peroxide in methanol to quench the endogenous peroxidase activity followed by incubation with $1 \%$ bovine serum albumin to block the nonspecific binding. The SCCA antibody (cat. no. ab154971, 1:100 dilution; Abcam, Cambridge, MA, USA) was incubated with the sections overnight at $4^{\circ} \mathrm{C}$. After washing, the tissue sections were treated with biotinylated secondary antibody (1:2,000; cat. no. 7074; Cell Signaling Technology, Inc., Danvers, MA, USA) for $60 \mathrm{~min}$ at room temperature. After rinsing with PBS, the slides were immersed for 2-5 min in DAB (3,3'-diaminobenzidine) (Sigma-Aldrich; Merck KGaA, Darmstadt, Germany) solution, 
Table I. Clinicopathological features of the papillary thyroid carcinoma (PTC) patients and SCCA expression.

\begin{tabular}{|c|c|c|c|c|c|}
\hline \multirow[b]{2}{*}{ Clinical features } & \multirow[b]{2}{*}{$\begin{array}{c}\text { No. of } \\
\text { patients }(\mathrm{N}=258)\end{array}$} & \multicolumn{2}{|c|}{ SCCA expression } & \multirow[b]{2}{*}{$\chi^{2}$} & \multirow[b]{2}{*}{ P-value } \\
\hline & & $\begin{array}{c}\text { Low }(\mathrm{n}=122) \\
\mathrm{n}(\%)\end{array}$ & $\begin{array}{c}\text { High }(\mathrm{n}=136) \\
\mathrm{n}(\%)\end{array}$ & & \\
\hline \multicolumn{6}{|l|}{ Sex } \\
\hline Male & 92 & $43(46.74)$ & $49(53.26)$ & \multirow[t]{2}{*}{0.017} & \multirow[t]{2}{*}{0.8956} \\
\hline Female & 166 & $79(47.59)$ & $87(52.41)$ & & \\
\hline \multicolumn{6}{|l|}{ Age, years } \\
\hline$<45$ & 106 & $52(49.06)$ & $54(50.94)$ & \multirow[t]{2}{*}{0.226} & \multirow[t]{2}{*}{0.6344} \\
\hline$\geq 45$ & 152 & $70(46.05)$ & $82(53.95)$ & & \\
\hline \multicolumn{6}{|l|}{ Tumor size (cm) } \\
\hline$\leq 4$ & 156 & $89(57.05)$ & $67(42.95)$ & \multirow[t]{2}{*}{19.09} & \multirow[t]{2}{*}{$0.0001^{\mathrm{a}}$} \\
\hline$>4$ & 102 & $33(32.35)$ & $69(67.65)$ & & \\
\hline \multicolumn{6}{|c|}{ Extrathyroid invasion } \\
\hline No & 127 & $72(56.69)$ & $55(43.31)$ & \multirow[t]{2}{*}{8.88} & \multirow[t]{2}{*}{$0.0029^{\mathrm{a}}$} \\
\hline Yes & 131 & $50(38.17)$ & $81(61.83)$ & & \\
\hline \multicolumn{6}{|c|}{ Lymph node metastasis (LNM) } \\
\hline No & 157 & $87(55.41)$ & $70(44.59)$ & \multirow[t]{2}{*}{10.36} & \multirow[t]{2}{*}{$0.0011^{\mathrm{a}}$} \\
\hline Yes & 101 & $35(34.65)$ & $66(65.35)$ & & \\
\hline \multicolumn{6}{|c|}{ Distant organ metastasis (DOM) } \\
\hline No & 187 & $97(51.87)$ & $90(48.13)$ & \multirow[t]{2}{*}{5.730} & \multirow[t]{2}{*}{$0.0167^{\mathrm{a}}$} \\
\hline Yes & 71 & $25(35.21)$ & $46(64.79)$ & & \\
\hline \multicolumn{6}{|l|}{ TNM stage } \\
\hline I-II & 120 & $69(57.50)$ & $51(42.50)$ & \multirow[t]{2}{*}{9.38} & \multirow[t]{2}{*}{$0.0022^{\mathrm{a}}$} \\
\hline III-IV & 138 & $53(38.41)$ & 85 (61.59) & & \\
\hline
\end{tabular}

SCCA, squamous cell carcinoma antigen; PTC, papillary thyroid carcinoma; TNM, tumor-node-metastasis. ${ }^{\text {a }}<0.01$, statistically significant difference.

then monitored under a microscope. The reaction was terminated with distilled water. Slides were then counterstained with hematoxylin, dehydrated and coverslipped.

IHC staining scores. The quantitative analysis of IHC staining was performed for SCCA protein expression levels in tissue specimens. Two experienced investigators scored independently all the slides using a method previously described (35). Scores considered both the proportion of positive-staining tumor cells and the staining intensity. The proportion of positively stained tumor cells was graded as follows: 0 (no positive tumor cells), 1 (10\% positive tumor cells), 2 ( $>10-50 \%$ positive tumor cells) and 3 ( $>50 \%$ positive tumor cells). The cells at each intensity of staining were recorded on a scale of 0 (no staining), 1 (weak staining, light yellow), 2 (moderate staining, yellowish brown) and 3 (strong staining, brown). The staining index (SI) was calculated as follows: $\mathrm{SI}=$ staining intensity $\mathrm{x}$ proportion of positively stained tumor cells. Using this method of assessment, we evaluated SCCA expression in tumor tissues by SI (scored as $0,1,2,3,4,6$ or 9). Cutoff values to define the high and low expression of SCCA were chosen based on a measure of heterogeneity with the log-rank test statistics with respect to OS. SCCA staining was quantified using a two-level grade system as follows: 0-3 indicates low expression and 4-9 indicates high expression. Mean optical density (MOD) represents the strength of staining signals by measuring per positive pixels.

Cell cultures and interference assays. K1 and 293T cell lines were obtained from the American Type Culture Collection (ATCC; Manassas, VA, USA) and maintained in RPMI-1640 medium (Invitrogen; Thermo Fisher Scientific, Inc., Waltham, MA, USA) supplementing with $10 \%$ fetal bovine serum (FBS) at $37^{\circ} \mathrm{C}$ with $5 \% \mathrm{CO}_{2}$ according to the manufacturer's instructions. Ectopic SCCA or Ras expression in the K1 cell line was achieved using retroviral vectors. Briefly, SCCA or Ras cDNA was cloned into retroviral transfer plasmid pMSCv to generate the pMSCv-SCCA or pMSCv-Ras expression vector, which was co-transfected in 293 T cells (ATCC) using standard calcium phosphate transfection method. Thirty-six hours after the co-transfection, supernatants were collected and incubated with the K1 cells for $24 \mathrm{~h}$ for the following assays. Knockdown SCCA or Ras cells were transfected with Lipofectamine 2000 (Invitrogen; Thermo Fisher Scientific, Inc.) using siRNA (Shanghai GenePharma Co., Ltd., Shanghai, China) according to the manufacturer's instructions. siRNA sequences of the 
sense strands were as follows: siSCCA-1, 5'-GCACAACAG ATTAAGAAGGTT-3' and siSCCA-2, 5'-CCGCTGTAGTAG GGATTCGGAT-3'; siRas-1, 5'-GGACGAAUAUGAUCCAAC ATT-3' and siRas-2, 5'-UGUUGGAUCAUAUUCGUCCTT-3'.

$q P C R$ assays. RNA was extracted using TRIzol reagent and a reverse transcription kit following the manufacturer's instructions (Invitrogen; Thermo Fisher Scientific, Inc.). PCR reactions were performed under the following conditions: Pre-denaturation at $94^{\circ} \mathrm{C}$ for $7 \mathrm{~min}$, denaturation at $94^{\circ} \mathrm{C}$ for $30 \mathrm{sec}$, annealing at $55^{\circ} \mathrm{C}$ for $30 \mathrm{sec}$, elongation at $72^{\circ} \mathrm{C}$ for $1 \mathrm{~min}$ and elongation at $72^{\circ} \mathrm{C}$ for $10 \mathrm{~min}$. The primers for quantitative real-time PCR assay were: SCCA, 5'-CATTTGTTT GCTGAAGCCACTAC-3' and 5'-CATGTTCGAAATCCA GTGATTCC-3'; GAPDH, 5'-AAGGTCGGAGTCAACGGA TTTG-3' and 5'-CCATGGGTGGAATCATATTGGAA-3. All primers were synthesized by Shanghai General Biotech, Co., Ltd. (Shanghai, China). GAPDH was used as an endogenous control. The $2^{-\triangle \Delta C q}$ method was used to quantify the relative mRNA amount (36).

Western blot assays. The samples were lysed in RIPA buffer consisting of $20 \mathrm{mM}$ Tris- $\mathrm{HCl}$ (pH 7.9), $137 \mathrm{mM} \mathrm{NaCl}, 5 \mathrm{mM}$ EDTA, $1 \mathrm{mM}$ EGTA, and $1 \%$ Triton X-100 that contained protease inhibitors for $20 \mathrm{~min}$ at $4^{\circ} \mathrm{C}$, and total protein concentration was measured using the BCA protein assay kit (Thermo Fisher Scientific, Inc.). Twenty micrograms of proteins was separated on a $10 \%$ SDS-PAGE and transferred onto polyvinylidene fluoride (PVDF) membranes (Roche Diagnostics, Indianapolis, IN, USA). Then, the membranes were blocked in 5\% skim milk in TBS-Tween buffer at room temperature for $2 \mathrm{~h}$ and incubated with a primary antibody against SCCA (dilution 1:1,000; cat. no. ab154971; Abcam), Ras (dilution 1:1,000; cat. no. 3339; Cell Signaling Technology, Inc.) or GAPDH (dilution 1:2,000; cat. no. ab9485; Abcam) at $4^{\circ} \mathrm{C}$ overnight. Then, the membranes were incubated with HRP-conjugated secondary antibodies (dilution 1:1,000; cat. no. 3056; Epitomics, Burlingame, CA, USA) at $4^{\circ} \mathrm{C}$ for $2 \mathrm{~h}$. The peroxidase activity was detected using Immobilon Western Chemiluminescent HRP Substrate (EMD Millipore, Billerica, MA, USA).

MTT assays. In brief, cell viability was evaluated in accordance with the MTT method. K1 cells were seeded in 96-well plates at $3 \times 10^{4}$ ectopic expressed cells $/ \mathrm{ml}$ and cultured for pre-selected time-points. Then, the cells were incubated with $10 \mu \mathrm{l}$ of $0.5 \%$ MTT solution for $4 \mathrm{~h}$ at $37^{\circ} \mathrm{C}$. The supernatant was discarded, and $150 \mu \mathrm{l}$ of dimethyl sulfoxide (DMSO) was added to each well. The 96-well plates were shaken for $10 \mathrm{~min}$ until the crystals dissolved completely. The absorbance was measured at a wavelength of $490 \mathrm{~nm}$ using a microplate reader (Bio-Rad Laboratories, Hercules, CA, USA).

Invasion and migration assays. Cells $\left(1 \times 10^{6}\right)$ were suspended in $200 \mu \mathrm{l}$ serum-free medium and seeded in the top chambers of 24-well Transwell plates (Corning Inc., Corning, NY, USA) coated with $30 \mu \mathrm{l}$ Matrigel (BD Biosciences, Franklin Lakes, NJ, USA). The bottom chambers of the Transwell plates were filled with $600 \mu 1$ medium containing $10 \%$ FBS. Cells were allowed to migrate for $48 \mathrm{~h}$ at $37^{\circ} \mathrm{C}$. After migration, cells in the top chambers were removed using a cotton swab, and the cells which migrated to the bottom chambers were fixed in $4 \%$ paraformaldehyde and stained with $0.1 \%$ crystal violet (Sigma-Aldrich; Merck KGaA). The fixed and stained cells were counted in three independent fields under a using an Olympus BX51 microscope at x 100 magnification (Olympus Corp., Tokyo, Japan). At least, three chambers were counted for each experiment. For the migration assay, a similar protocol was followed except for the replacement of the top chamber of the Transwell plate with an uncoated chamber. The bottom chamber was filled with $700 \mu \mathrm{l}$ medium containing $10 \%$ FBS, and cells were allowed to migrate for $24 \mathrm{~h}$.

Xenograft tumor experiments. K1 cells $\left(1 \times 10^{7}\right)$ with ectopic SSCA expression were injected into the mammary fat pads of 6- to 8-week-old athymic female nude mice purchased from the Shanghai Experimental Animal Center (Shanghai, China). Nine mice weighing 23-25 g were used, and they were maintained under SPF conditions at $20-26^{\circ} \mathrm{C}$, relative humidity $40-70 \%$ and a 12-h light-dark cycle. All food was treated with high temperature steam disinfection $\left(60 \mathrm{~min}, 120^{\circ} \mathrm{C}\right)$. All water was acidified by hydrochloric acid and adjusted to a pH between 2.5 and 2.8. All efforts were made to minimize animal suffering. Tumor growth and progression were monitored for more than 9 weeks. Tumor size was measured once a week by calipers, and tumor volume was calculated as: Volume $\left(\mathrm{mm}^{3}\right)=$ length $\mathrm{x}$ width ${ }^{2} \mathrm{x} 0.5$. At the end of the experiment, animals were euthanized by $\mathrm{CO}_{2}$ asphyxiation, and tumors were carefully resected, weighed and stored for analysis.

Statistical analysis. Statistical analyses were performed with GraphPad Prism 5.0 (GraphPad Software, Inc., La Jolla, CA, USA). The Chi-squared test was used to analyze the associations between SCCA expression and clinicopathological factors. Kaplan-Meier analysis was used for overall survival (OS), disease-free survival (DFS), lymph node recurrence-free survival (LNRFS) and distant recurrence-free survival (DRFS) calculations. Cox regression analysis determined the prognostic significance and pathologic characteristics. The correlation between SCCA and Ras mRNA expression was determined using the Pearson's correlation test. The comparison of two independent groups was analyzed using Student's t-tests. Multiple group comparisons were analyzed with one-way ANOVA and Tukey's post hoc test. All statistical tests were two-tailed, and P-values $<0.05$ were considered statistically significant.

\section{Results}

SCCA protein expression correlates with tumor stage and metastasis in the PTC patient samples. A total of 258 postoperative samples obtained from patients diagnosed with different stages of PTC, based on the TNM staging system (37), were analyzed: Stage I $(n=52)$, stage II $(n=68)$, stage III $(n=79)$ and stage IV $(n=59)$. The clinical features of the patients with high and low levels of SCCA expression are summarized in Table I. Low SCCA expression was observed in $47.29 \%(122 / 258)$ of the samples, while $52.71 \%(136 / 258)$ showed high SCCA expression levels. In tumors $>4 \mathrm{~cm}, 67.65 \%$ (69/102) of the samples showed high SCCA expression, while only $32.35 \%$ 


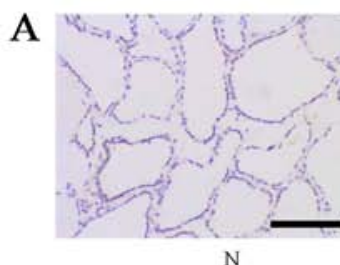

B

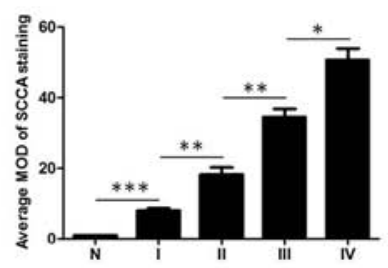

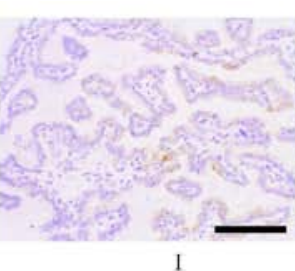

C

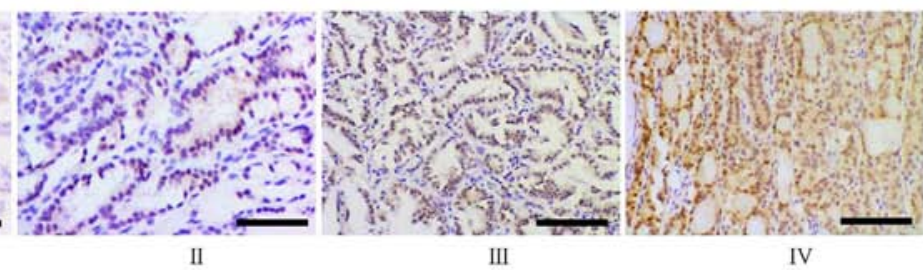

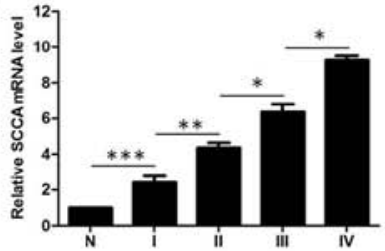

D

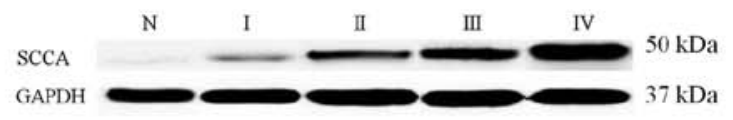

E

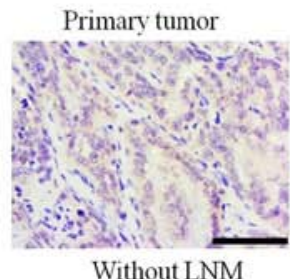

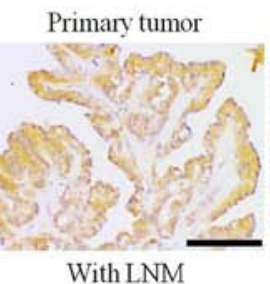

With LNM

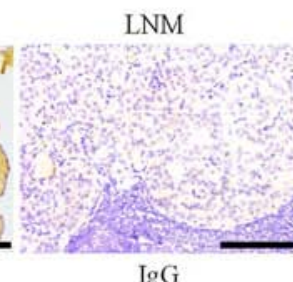

IgG

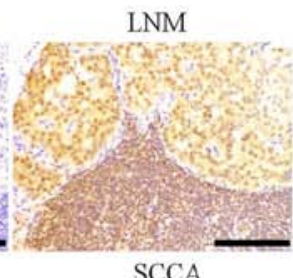

SCCA

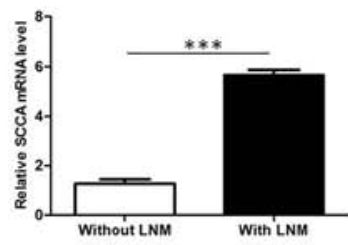

Figure 1. SCCA expression levels are significantly correlated with human PTC metastasis and progression. (A) Representative images from IHC assays of paraffin-embedded specimens of different stages of PTC and non-cancerous tissues. Stage I ( $n=52)$, stage II $(n=68)$, stage III $(n=79)$, stage IV ( $n=59)$ and non-cancerous $(\mathrm{N})$ tissues $(\mathrm{n}=28)$ (IHC, magnification x200, scale bars, $100 \mu \mathrm{m}$ ). (B) SCCA expression is quantified as the mean optical density (MOD) for SCCA staining among non-cancerous tissues and various PTC stages $\left({ }^{*} \mathrm{P}<0.05,{ }^{* * *} \mathrm{P}<0.01,{ }^{* * * *} \mathrm{P}<0.001\right.$, one-way ANOVA). (C) SCCA mRNA expression levels in non-cancerous tissues and PTC stages I to IV using qRT-PCR $\left({ }^{*} \mathrm{P}<0.05,{ }^{* *} \mathrm{P}<0.01,{ }^{* * *} \mathrm{P}<0.001\right.$, one-way ANOVA). (D) SCCA protein expression levels in non-cancerous tissues and PTC stages I to IV using western blot assay. (E) IHC staining in primary PTC without lymph node metastasis (LNM), PTC with LNM and metastasis lymph node (IHC, magnification x 200, scale bars, $100 \mu \mathrm{m} ;{ }^{* * *} \mathrm{P}<0.001$, Student's t-tests). SCCA, squamous cell carcinoma antigen; PTC, papillary thyroid carcinoma; IHC, immunohistochemistry.

(33/102) of tumors showed low SCCA expression. Moreover, more than $50 \%$ of patients $(131 / 258)$ showed extra thyroid invasion. Moreover, $39.15 \%$ of patients (101/258) presented with lymph node metastasis (LNM), and $27.52 \%$ of patients (71/258) had distant organ metastasis (DOM). Based on the TNM staging system, $46.51 \%$ of patients (120/258) presented with a stage I+II disease, and $53.49 \%$ of patients (138/258) with a stage III+IV disease.

As summarized in Table I, there was a significant correlation between high SCCA expression and tumor size $(\mathrm{P}=0.0001)$, extrathyroid invasion $(\mathrm{P}=0.0029)$, lymph node metastasis $(\mathrm{P}=0.0011)$, distant organ metastasis $(\mathrm{P}=0.0167)$, and TNM stage $(\mathrm{P}=0.0022)$. These results indicate that SCCA expression is significantly correlated with aggressive metastasis and progression in PTC.

Next, we explored whether SCCA expression levels are correlated with tumor histologic features, by examining 28 non-cancerous tissues and 258 PTC tissues, including 52 stage I, 68 stage II, 79 stage III and 59 stage IV tissues, by IHC. SCCA expression in non-cancerous tissues was low or non-detected. In contrast, SCCA protein was highly expressed in higher stages of PTC, compared with non-cancerous tissues (Fig. 1A). In addition, SCCA expression increased as the stage of PTC increased. The mean optical density (MOD) of SCCA staining among non-cancerous tissues and PTC specimens of different stages is documented in Fig. 1B. Stronger SCCA staining was observed as the PTC malignant stage degree increased $(\mathrm{P}<0.05)$. In agreement with the IHC results, SCCA mRNA levels, as determined by qRT-PCR assays, also increased and were higher as the malignant degree increased $(\mathrm{P}<0.05$; Fig. 1C). To validate these findings, we examined SCCA protein expression levels in PTC stages I to IV by immunoblotting. Compared with lower stage PTC, SCCA protein expression was markedly strong in higher stage tumors (Fig. 1D). Noteworthy, there was a significant correlation between high SCCA expression and lymph node metastasis, and in most cases a strong SCCA staining was correlated with tumors with lymph node metastasis (Fig. 1E). Moreover, the primary PTC with LNM showed high SCCA mRNA level, compared with the tumors without LNM $(\mathrm{P}<0.001)$. Collectively, our findings indicate that higher SCCA expression levels are associated with PTC stage and metastasis.

SCCA expression and patient survival. We next investigated whether a correlation exists between SCCA levels in PTC and patient survival, using Kaplan-Meier analysis. During the 8-year patient follow-up, the overall survival (OS) rate was (119/136) $87.5 \%$ in the high SCCA expression group, but it was (117/122) 95.9\% in the low SCCA expression group ( $\mathrm{P}=0.0092$; Fig. 2A). Our results also indicate that SCCA overexpression was significantly correlated with low disease-free survival (DFS) ( $\mathrm{P}=0.0007$; Fig. 2B). The prognostic value of SCCA expression was also estimated in the 

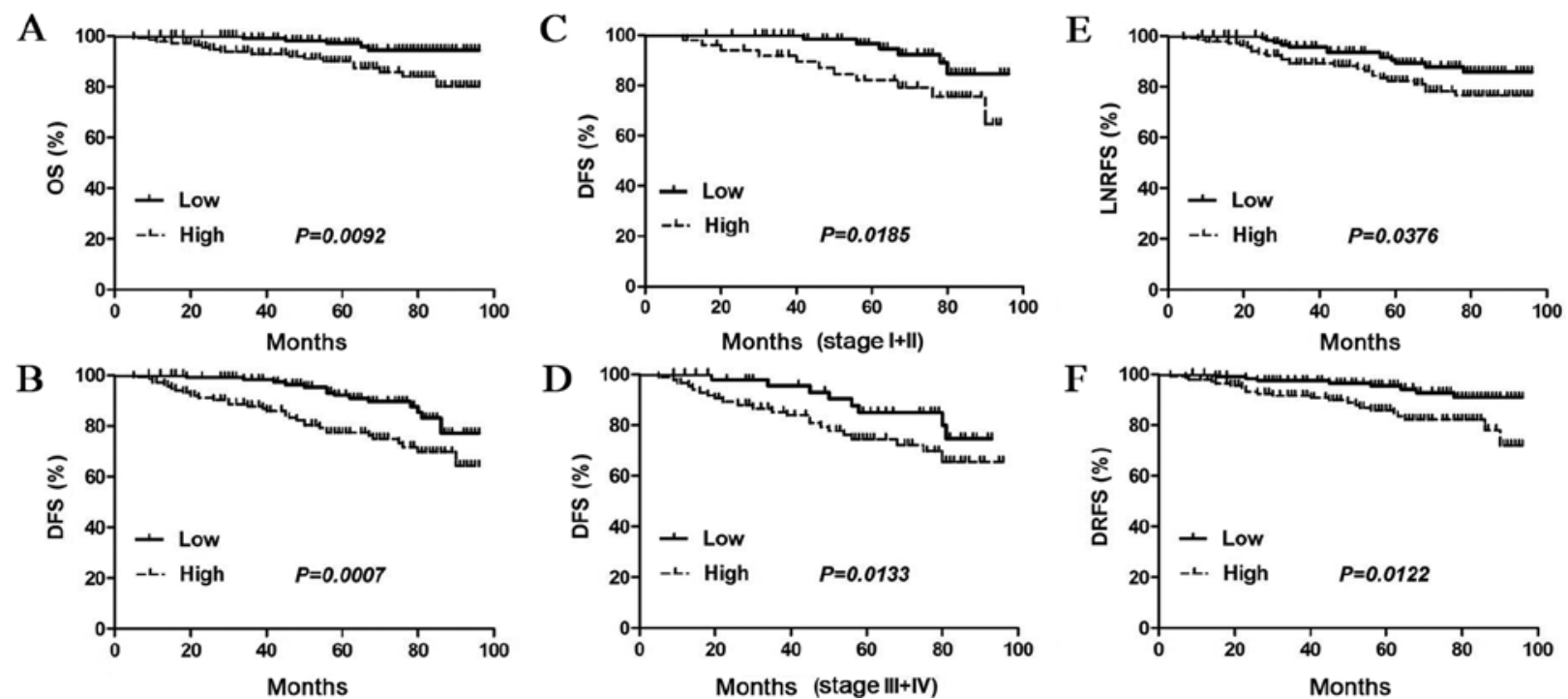

Figure 2. Correlation between SCCA expression and survival in PTC patients. (A and B) SCCA overexpression correlates with a reduced overall survival $(\mathrm{OS})$ and disease-free survival (DFS) $(\mathrm{P}<0.01)$. (C and D) DFS in PTC patients with high SCCA expression is significantly reduced, compared to those with low expression in either stage I+II subgroup $(\mathrm{P}=0.0185)$ or stage III+IV subgroup $(\mathrm{P}=0.0133)$. ( $\mathrm{E}$ and $\mathrm{F}) \mathrm{SCCA}$ overexpression in $\mathrm{PTC}$ patients is significantly associated with lymph node recurrence-free survival (LNRFS; $\mathrm{P}=0.0376$ ) and distant recurrence free survival (DRFS; $\mathrm{P}=0.0122$ ). SCCA, squamous cell carcinoma antigen; PTC, papillary thyroid carcinoma.

Table II. Univariate regression analysis of disease-free survival (DFS) in the papillary thyroid carcinoma (PTC) cases.

\begin{tabular}{|c|c|c|}
\hline \multirow[b]{2}{*}{ Clinical features } & \multicolumn{2}{|c|}{ PTC $(\mathrm{N}=258)$} \\
\hline & $\mathrm{HR}(95 \% \mathrm{CI})$ & P-value \\
\hline Age, years & & 0.651 \\
\hline$<45$ & 1 & \\
\hline$\geq 45$ & $1.975(1.546-2.372)$ & \\
\hline Tumor size, $\mathrm{cm}$ & & $0.003^{\mathrm{a}}$ \\
\hline$\leq 4$ & 1 & \\
\hline$>4$ & $1.352(0.926-3.356)$ & \\
\hline $\begin{array}{l}\text { Lymph node metastasis } \\
\text { (LNM) }\end{array}$ & & $0.001^{\mathrm{a}}$ \\
\hline No & 1 & \\
\hline Yes & $3.239(1.651-5.563)$ & \\
\hline $\begin{array}{l}\text { Distant organ metastasis } \\
\text { (DOM) }\end{array}$ & & $0.012^{\mathrm{a}}$ \\
\hline No & 1 & \\
\hline Yes & $1.225(0.367-2.328)$ & \\
\hline Extrathyroid invasion & & $0.018^{\mathrm{a}}$ \\
\hline No & 1 & \\
\hline Yes & $1.308(0.563-3.265)$ & \\
\hline SCCA expression & & $<0.001^{\mathrm{a}}$ \\
\hline Low & 1 & \\
\hline High & $2.879(0.432-6.395)$ & \\
\hline TNM stage & & $0.002^{\mathrm{a}}$ \\
\hline I-II & 1 & \\
\hline III-IV & $2.331(1.247-5.762)$ & \\
\hline
\end{tabular}

SCCA, squamous cell carcinoma antigen; PTC, papillary thyroid carcinoma; HR, hazard ratio; $\mathrm{CI}$, confidence interval. ${ }^{\mathrm{a}} \mathrm{P}<0.05$, statistically significant difference. various subgroups of PTC patients, based on the TNM stage. In agreement with our previous observations, DFS in PTC patients with high SCCA expression was significantly reduced, compared to those with low SCCA expression in either stages I+II subgroup ( $\mathrm{P}=0.0185$; Fig. $2 \mathrm{C}$ ) or stages III+IV subgroup $(\mathrm{P}=0.0133$; Fig. $2 \mathrm{D})$.

Since tumor metastasis plays an important role in poor prognosis and reduced survival, the correlation was assessed between SCCA expression and lymph node recurrence-free survival (LNRFS) and distant recurrence-free survival (DRFS), by the Kaplan-Meier method. We observed that SCCA expression was significantly associated with LNRFS ( $\mathrm{P}=0.0376$; Fig. 2E) and DRFS ( $\mathrm{P}=0.0122$; Fig. 2F).

To determine whether SCCA expression could be regarded as an independent prognostic factor, a Cox regression analysis for DFS was performed (Table II). Moreover, univariate analysis of the clinical features revealed significant correlation between DFS and tumor size $(\mathrm{P}=0.003)$, lymph node metastasis $(\mathrm{LNM})(\mathrm{P}=0.001)$, distant organ metastasis (DOM) $(\mathrm{P}=0.012)$, extrathyroid invasion $(\mathrm{P}=0.018)$, SCCA expression $(\mathrm{P}<0.001)$ and TNM stage $(\mathrm{P}=0.002)$. Additionally, using a multivariate analysis, we observed significant relationships between $\mathrm{DFS}$ and tumor size $(\mathrm{P}=0.015), \mathrm{LNM}(\mathrm{P}=0.001)$, DOM $(\mathrm{P}=0.026)$, extrathyroid invasion $(\mathrm{P}=0.021)$, SCCA expression $(\mathrm{P}<0.001)$ and TNM stage $(\mathrm{P}=0.003$; Table III $)$, which were regarded as significant prognostic factors for PTC patients. Taken together, our findings indicate that SCCA protein may be considered as a predictive prognostic factor in PTC patients.

SCCA promotes PTC growth in vitro and in vivo. To evaluate whether SCCA plays an important role in tumor growth, we performed SCCA overexpression and knockdown assays using plasmid and specific siRNAs (Fig. 3A). SCCA overexpression significantly promoted K1 thyroid cancer cell proliferation. In contrast, SCCA knockdown significantly 
A

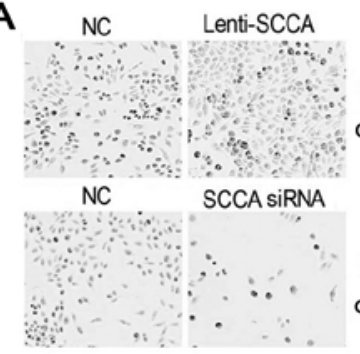

D

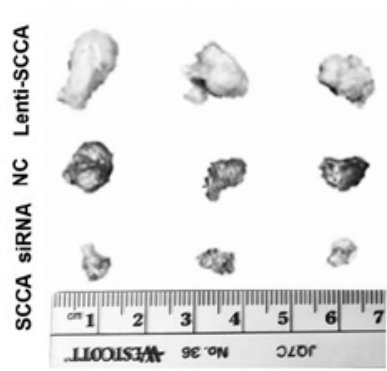

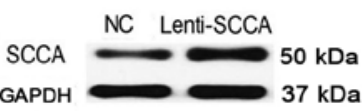

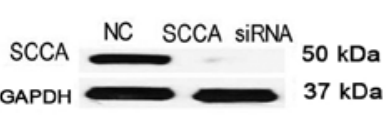

B

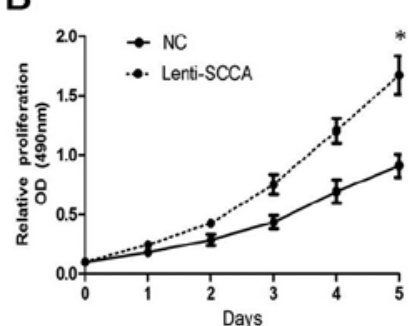

C

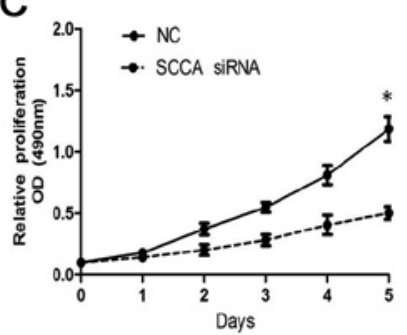

$\mathrm{F}$
E

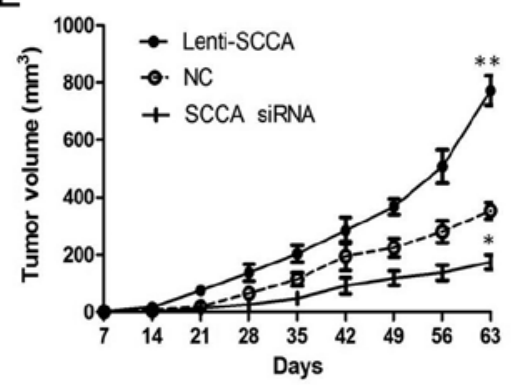

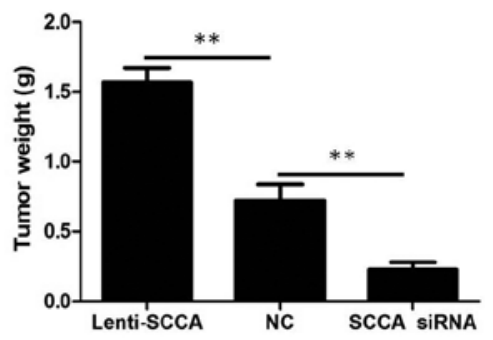

Figure 3. SCCA promotes PTC growth in vitro and in vivo. (A) Representative images by $0.1 \%$ crystal violet staining and western blotting images for SCCA protein from thyroid K1 cells with overexpressed or knockdown SCCA for $48 \mathrm{~h}$. (B and C) SCCA overexpression significantly promoted K1 thyroid cancer cell proliferation, and SCCA knockdown markedly inhibited cell proliferation as demonstrated using MTT assays ('P<0.05, Student's t-tests). (D) Representative images of the tumors after 9 weeks of implantation. (E) SCCA knockdown reduced the volume of xenograft tumors, while SCCA overexpression accelerated tumor growth $\left({ }^{*} \mathrm{P}<0.05,{ }^{* *} \mathrm{P}<0.01\right.$, one-way ANOVA). (F) SCCA knockdown decreased the weight of xenograft tumors, while SCCA overexpression significantly increased tumor weight $\left({ }^{* *} \mathrm{P}<0.01\right.$, one-way ANOVA). SCCA, squamous cell carcinoma antigen; PTC, papillary thyroid carcinoma.

Table III. Multivariate regression analysis of disease-free survival (DFS) in the papillary thyroid carcinoma (PTC) cases.

\begin{tabular}{|c|c|c|}
\hline \multirow[b]{2}{*}{ Clinical features } & \multicolumn{2}{|l|}{ PTC (N=258) } \\
\hline & HR $(95 \%$ CI) & P-value \\
\hline Tumor size, $\mathrm{cm}$ & & $0.015^{\mathrm{a}}$ \\
\hline$\leq 4$ & 1 & \\
\hline$>4$ & $1.326(0.128-3.876)$ & \\
\hline $\begin{array}{l}\text { Lymph node metastasis } \\
\text { (LNM) }\end{array}$ & & $0.001^{\mathrm{a}}$ \\
\hline No & 1 & \\
\hline Yes & $2.213(1.868-5.387)$ & \\
\hline $\begin{array}{l}\text { Distant organ metastasis } \\
\text { (DOM) }\end{array}$ & & $0.026^{\mathrm{a}}$ \\
\hline No & 1 & \\
\hline Yes & $1.653(0.238-3.552)$ & \\
\hline Extrathyroid invasion & & $0.021^{\mathrm{a}}$ \\
\hline No & 1 & \\
\hline Yes & $1.763(0.342-3.652)$ & \\
\hline SCCA expression & & $<0.001^{\mathrm{a}}$ \\
\hline Low & 1 & \\
\hline High & $3.487(1.863-6.238)$ & \\
\hline TNM stage & & $0.003^{\mathrm{a}}$ \\
\hline I-II & 1 & \\
\hline III-IV & $2.365(1.339-5.382)$ & \\
\hline
\end{tabular}

SCCA, squamous cell carcinoma antigen; PTC, papillary thyroid carcinoma; HR, hazard ratio; $\mathrm{CI}$, confidence interval. ${ }^{\mathrm{a}} \mathrm{P}<0.05$, statistically significant difference. inhibited cell proliferation as demonstrated using MTT assays in vitro $(\mathrm{P}<0.05$; Fig. $3 \mathrm{~B}$ and $\mathrm{C})$. To assess whether SCCA was involved in PTC growth in vivo, we performed a tumor xenograft model using K1 cells, expressing different levels of SCCA protein. Representative images of the xenograft tumors at 9 weeks are shown (Fig. 3D). Nine weeks following implantation, tumors in which SCCA was knocked-down showed a reduced xenograft volume, while tumors with overexpressed SCCA had accelerated growth, compared to the control $(\mathrm{P}<0.05$; Fig. 3E). In agreement, SCCA knockdown decreased tumor weight, while SCCA overexpression significantly increased tumor weight $(\mathrm{P}<0.01$; Fig. $3 \mathrm{~F})$. Taken together, our data indicate that SCCA knockdown inhibits tumor growth, suggesting that SCCA protein could represent a target for PTC therapy.

SCCA promotes thyroid cancer cell Kl invasiveness in vitro. Tumor invasiveness is significantly correlated with aggressive metastasis (6). To determine whether the SCCA protein plays an important role in tumor invasion, we performed migration and invasion assays in $\mathrm{K} 1$ cells with overexpressed or silenced SCCA. Overexpression of SCCA significantly increased K1 thyroid cancer cell migration, while SCCA knockdown significantly reduced the migratory capability of the $\mathrm{K} 1$ cells $(\mathrm{P}<0.05$; Fig. 4A and B). In addition, SCCA overexpression significantly promoted the invasive capability of K1 thyroid cancer cells, while SCCA knockdown significantly inhibited the invasion of $\mathrm{K} 1$ cells $(\mathrm{P}<0.05$; Fig. $4 \mathrm{C}$ and $\mathrm{D})$. Collectively, these data suggest that SCCA protein may be associated with tumor metastasis.

Ras/SCCA pathway activation enhances tumor proliferation and invasion. Genetic alterations in the mitogen-associated 

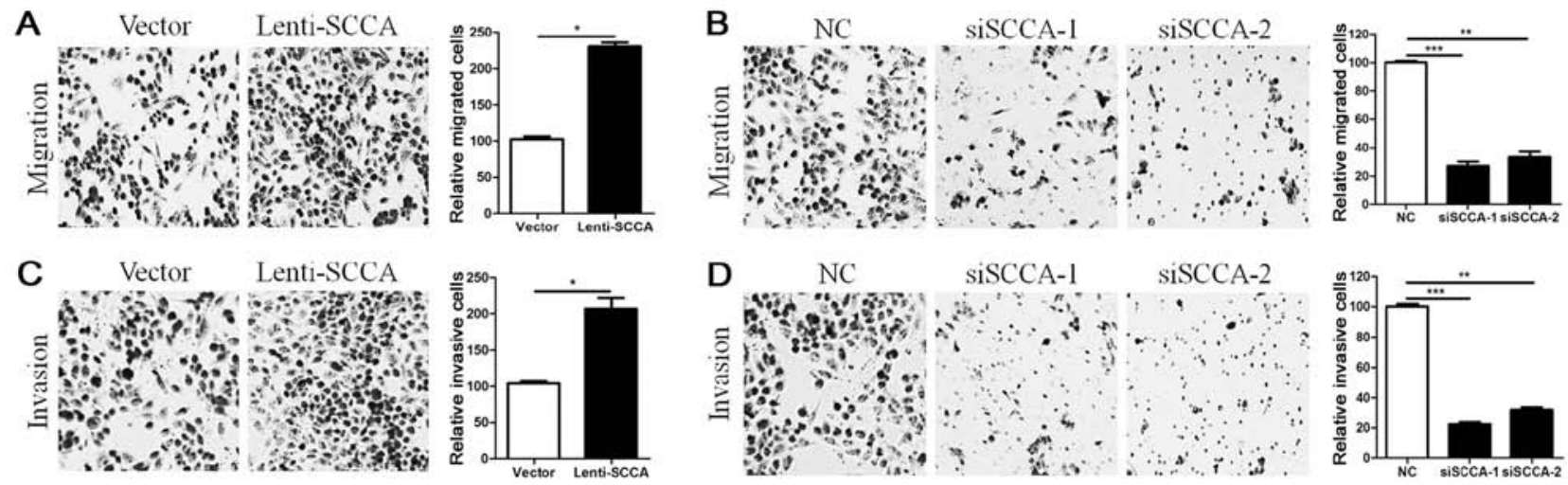

Figure 4. SCCA promotes the migration and invasion of K1 thyroid cancer cells. (A and B) SCCA overexpression significantly increased K1 thyroid cancer cell migration, while SCCA knockdown reduced cell migratory capability. (C and D) SCCA overexpression markedly promoted the invasive capability of K1 thyroid cancer cells, while SCCA knockdown significantly inhibited cell invasion. $\left({ }^{*} \mathrm{P}<0.05,{ }^{* *} \mathrm{P}<0.01,{ }^{* * *} \mathrm{P}<0.001\right.$, Student's t-tests or one-way ANOVA). SCCA, squamous cell carcinoma antigen.

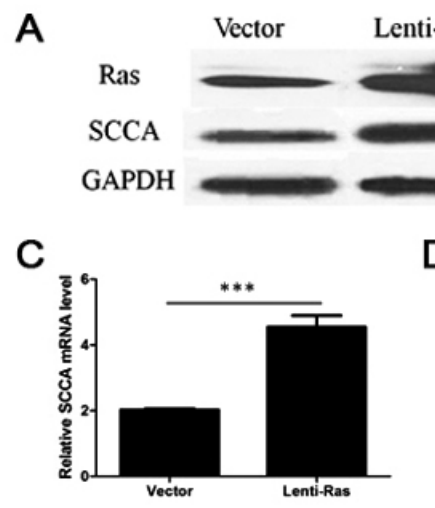

G

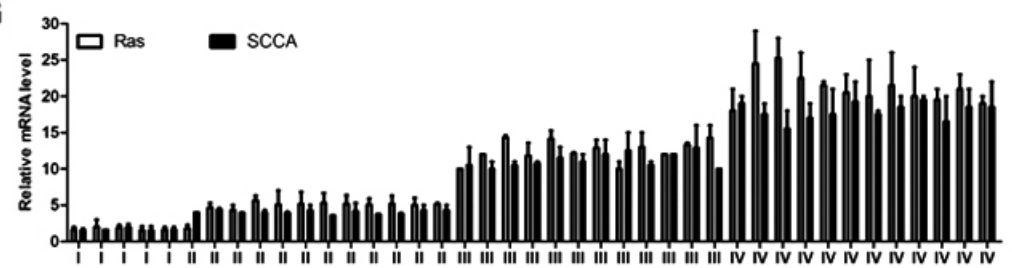

B

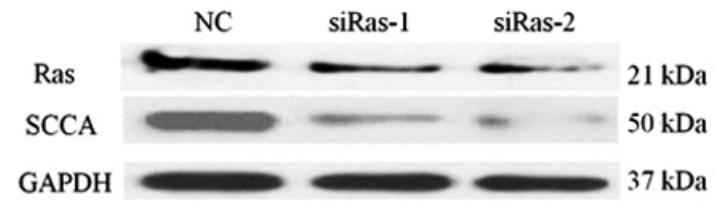

E
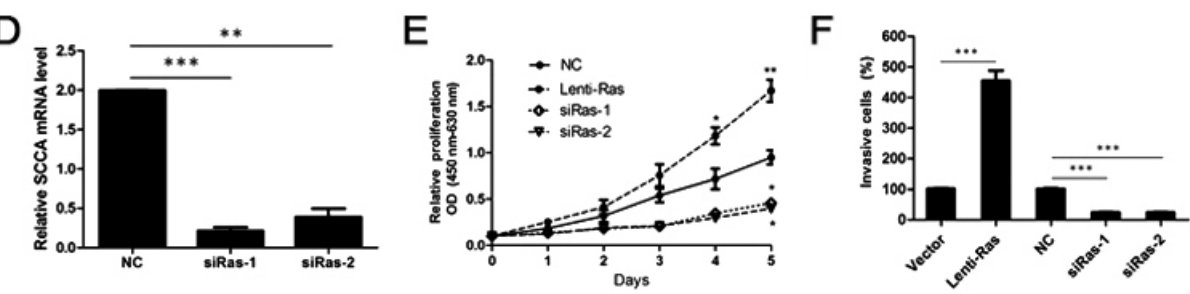

$\mathrm{H}$

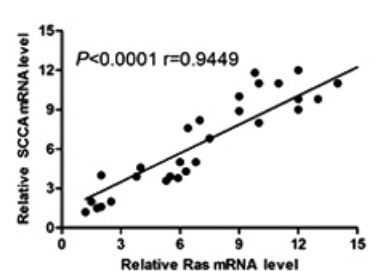

Figure 5. Ras upregulates SCCA protein and promotes PTC growth and invasion. (A and B) Ras overexpression significantly increased SCCA protein levels, whereas Ras knockdown markedly reduced SCCA expression. (C and D) Ras overexpression increased SCCA mRNA levels, while Ras knockdown significantly decreased SCCA mRNA levels $\left({ }^{* *} \mathrm{P}<0.01,{ }^{* * *} \mathrm{P}<0.001\right.$, Student's t-tests or one-way ANOVA). (E and F) Ras overexpression significantly promoted K1 cell proliferation and invasion, while Ras knockdown significantly inhibited cell proliferation and invasion in vitro $\left({ }^{*} \mathrm{P}<0.05,{ }^{* *} \mathrm{P}<0.01,{ }^{* * * *} \mathrm{P}<0.001\right.$, one-way ANOVA). (G) Ras and SCCA mRNA expression increased from stage I to IV in PTC tissues with a similar tendency by qRT-PCR, including 5 stage I, 12 stage II, 12 stage III and 12 stage IV samples. (H) SCCA expression is positively correlated with Ras levels in PTC tissues $(\mathrm{n}=41, \mathrm{r}=0.9449$, P<0.0001). SCCA, squamous cell carcinoma antigen; PTC, papillary thyroid carcinoma.

protein kinase (MAPK) pathway play an important role in the progression of PTC (29). In addition, BRAF point mutations, RET/PTC oncogene rearrangements, and RAS point mutations are frequent in advanced PTC and are associated with poor prognosis $(6,29,38)$. Previously, it was shown that Ras activation upregulated SCCA protein levels in colon cancer cell lines (34). However, whether Ras upregulates SCCA protein in PTC remains unclear. For this reason, to determine whether Ras upregulates SCCA protein to promote PTC progression, we analyzed SCCA expression using an RAS overexpression plasmid and specific siRas in vitro. Ras overexpression significantly increased SCCA protein level, while Ras silencing markedly decreased the SCCA protein level (Fig. 5A and B). In concordance, knockdown of Ras markedly decreased SCCA mRNA levels, while Ras overexpression increased the mRNA level of SCCA ( $P<0.01$; Fig. 5C and D). Moreover, Ras overexpression significantly enhanced K1 thyroid cancer cell proliferation and invasion in vitro, while knockdown of Ras significantly suppressed cell proliferation and invasion $(\mathrm{P}<0.01$; Fig. 5E and $\mathrm{F})$. Noteworthy, Ras and SCCA mRNA expression levels increased from stage I to IV with similar style in PTC tissues by qRT-PCR assay (Fig. 5G). Moreover, SCCA mRNA levels were significantly correlated with Ras levels in PTC tissues (Fig. 5H, r=0.9449, P<0.0001). Taken together, these results indicate that Ras could upregulate SCCA expression to promote PTC proliferation and invasion. 


\section{Discussion}

PTC, the most common cancer of the endocrine system, accounts for the highest number of thyroid cancer cases diagnosed in recent decades (39). Even though PTC is usually curable and has a 5-year survival rate higher than $95 \%$, in some circumstances, PTC de-differentiates and becomes aggressive, resulting in a poor prognosis. Therefore, novel PTC biomarkers are needed to better predict patient prognosis and to facilitate the development of personalized therapies for PTC patients. In the present study, we identified SCCA as a critical growth regulator and a target marker of PTC.

SCCA protein is a member of the serine protease inhibitor family of proteins. Initially identified as an important marker for advanced carcinomas of the cervix (18), SCCA has now been shown to have a critical function in many cancer types, including breast (11), liver $(12,16)$, lung $(13)$, head and neck carcinoma (14), and esophageal cancers (15). In agreement, we observed that SCCA levels were significantly overexpressed in PTC, and were correlated with the clinicopathological features of PTC. In agreement with previous research, SCCA overexpression was associated with high-grade breast carcinoma and was correlated with estrogen receptor/progesterone receptor double-negative tumors as well as with a poor prognosis for breast cancer patients (34).

To directly evaluate whether SCCA is critical to tumor growth, we performed a genetic approach to knockdown and overexpress SCCA in PTC cells. SCCA knockdown markedly inhibited the proliferation of $\mathrm{K} 1$ thyroid cancer cells, while SCCA overexpression accelerated xenograft tumor growth. Furthermore, SCCA overexpression significantly increased K1 thyroid cancer cell migration and invasion, while SCCA suppression reduced the migratory and invasive capability of $\mathrm{K} 1$ cells. Similar observations have been described in breast and cervical cancers, where SCCA was associated with both advanced stage and strong invasive breast carcinoma (11), and also positively correlated with para-aortic lymph node metastasis in cervical cancer (40). Moreover, SCCA levels have also been shown to coincide to a degree with tumor infiltration and frequency of lymph node metastasis in both cervical and esophageal squamous cell carcinomas $(41,42)$. Mechanistically, it has been shown that SCCA increased tumor invasiveness by promoting oncogenic transformation and epithelial-mesenchymal transition (EMT) in breast cancer (43). Collectively, these findings support a role for SCCA in PTC growth and invasiveness.

Lymph node metastasis is one of the most important clinical features in treatment determination and prognosis for cancer patients, including PTC. In PTC, despite being highly curable and presenting a 10-year survival rate of more than $90 \%$, lymph node metastasis, especially in the neck, occurs in $20-50 \%$ of all tumor patients and regional recurrence was observed in $5-20 \%$ of patients who underwent total thyroidectomy (2). Our findings indicate a significant correlation between high SCCA expression and lymph node metastasis, and in most cases we observed a strong SCCA staining in primary tumors with lymph node metastasis. In agreement, lymph node metastasis has been shown to be the main factor affecting the 5-year overall survival rate of cervical cancer patients (44). The prognosis of cervical carcinoma with common iliac lymph node metastasis was even poorer, with the 5-year overall survival rate of 25-47.8\% (45). Moreover, the primary PTC with LNM showed a higher SCCA mRNA level compared with the tumors without LNM, suggesting that SCCA overexpression could be associated with PTC stage and metastasis. Previous observation also suggested that the SCCA level in serum is a prediction factor for lymph node metastasis (46).

The Ras signaling pathway, a highly complex signaling pathway, regulates tumor initiation and progression. Recent evidence supports the notion that Ras oncoproteins participate in the acquisition of tumor cells with EMT plasticity, enhanced metastatic potential and poor patient survival (47). In thyroid cancer, BRAF and Ras mutations as well as RET rearrangements recently have been given a great deal of attention as novel prognostic markers for thyroid carcinoma. Ras mutations seem to favorably activate the PI3K/AKT pathway in thyroid tumorigenesis, as suggested by the preferential association of RAS mutations with AKT phosphorylation in thyroid cancers $(38,48)$. We observed that Ras overexpression significantly increased SCCA protein levels, while Ras knockdown markedly decreased SCCA protein levels. Noteworthy, Ras and SCCA mRNA expression levels increased from stage I to IV with similar style in PTC tissues. Furthermore, SCCA mRNA levels were significantly associated with the Ras levels in PTC tissues. These results indicate that Ras enhances SCCA expression to promote PTC proliferation and invasion.

In conclusion, our results indicate a critical role for SCCA in PTC growth and invasion. SCCA protein is overexpressed in PTC tissues, correlating with clinical stage and metastasis in PTC. SCCA protein may be a predictive prognostic factor in PTC patients. Moreover, activation of the Ras/SCCA pathway plays an important role in promoting cancer cell proliferation and metastasis in PTC.

\section{Acknowledgements}

Not applicable.

\section{Funding}

The present study was supported by grants from the National Natural Science Foundation of China (no. 81702654) and the Natural Science Foundation of Guangdong Province (no. 2017A030313642).

\section{Availability of data and materials}

The datasets used during the present study are available from the corresponding author upon reasonable request.

\section{Authors' contributions}

DL, YL and SL conceived the research project, designed the experiments and edited the manuscript. DL, WZ, GGM, HL and $\mathrm{NO}$ edited the manuscript and provided overall support for the experimental process. DL, LT, ZD and XP performed the experiments and collected the clinical samples. DL, ML and YZ analyzed the results and conducted the statistical analysis of data. DL, YL and GGM wrote and revised the manuscript. 
All authors read and approved the manuscript and agree to be accountable for all aspects of the research in ensuring that the accuracy or integrity of any part of the work are appropriately investigated and resolved.

\section{Ethics approval and consent to participate}

The present study was performed in accordance with the policies of the Institutional Research Ethics Committee of Sun Yat-Sen Memorial Hospital. Written informed consent was obtained from the study participants at the Sun Yat-Sen Memorial Hospital of Guangzhou City. All animal experiments were carried out under the guidelines of the Sun Yat-Sen University Committee for Use and Care of Laboratory Animals and approved by the Animal Experimentation Ethics Committee of Sun Yat-Sen University.

\section{Patient consent for publication}

Not applicable.

\section{Competing interests}

The authors declare that they have no conflict of interest.

\section{References}

1. Kim MJ, Won JK, Jung KC, Kim JH, Cho SW, Park DJ and Park YJ: Clinical characteristics of subtypes of follicular variant papillary thyroid carcinoma. Thyroid 28: 311-318, 2018.

2. Sipos JA and Mazzaferri EL: Thyroid cancer epidemiology and prognostic variables. Clin Oncol (R Coll Radiol) 22: 395-404, 2010.

3. Zheng R, Zeng H, Zhang S and Chen W: Estimates of cancer incidence and mortality in China, 2013. Chin J Cancer 36: 66, 2017.

4. Schmidbauer B, Menhart K, Hellwig D and Grosse J: Differentiated thyroid cancer-treatment: State of the art. Int J Mol Sci 18: pii: E1292, 2017.

5. Nasef HO, Nixon IJ and Wreesmann VB: Optimization of the risk-benefit ratio of differentiated thyroid cancer treatment. Eur J Surg Oncol 44: 276-285, 2018.

6. Chmielik E, Rusinek D, Oczko-Wojciechowska M, Jarzab M, Krajewska J, Czarniecka A and Jarzab B: Heterogeneity of thyroid cancer. Pathobiology 85: 117-129, 2018.

7. Kato H,MoriokaH, Aramaki S and Torigoe T: Radioimmunoassay for tumor-antigen of human cervical squamous cell carcinoma Cell Mol Biol Incl Cyto Enzymol 25: 51-56, 1979.

8. Schneider SS, Schick C, Fish KE, Miller E, Pena JC, Treter SD, Hui SM and Silverman GA: A serine proteinase inhibitor locus at $18 \mathrm{q} 21.3$ contains a tandem duplication of the human squamous cell carcinoma antigen gene. Proc Natl Acad Sci USA 92: 3147-3151, 1995.

9. Vassilakopoulos T, Troupis T, Sotiropoulou C, Zacharatos P, Katsaounou P, Parthenis D, Noussia O, Troupis G, Papiris S, Kittas C, et al: Diagnostic and prognostic significance of squamous cell carcinoma antigen in non-small cell lung cancer. Lung Cancer 32: 137-144, 2001.

10. Guido M, Roskams T, Pontisso P, Fassan M, Thung SN, Giacomelli L, Sergio A, Farinati F, Cillo U and Rugge M: Squamous cell carcinoma antigen in human liver carcinogenesis. J Clin Pathol 61: 445-447, 2008.

11. Catanzaro JM, Guerriero JL, Liu J, Ullman E, Sheshadri N, Chen JJ and Zong WX: Elevated expression of squamous cell carcinoma antigen (SCCA) is associated with human breast carcinoma. PLoS One 6: e19096, 2011.

12. Pontisso P: Role of SERPINB3 in hepatocellular carcinoma. Ann Hepatol 13: 722-727, 2014.

13. Zhao W, Yu H, Han Z, Gao N, Xue J and Wang Y: Clinical significance of joint detection of serum CEA, SCCA, and bFGF in the diagnosis of lung cancer. Int J Clin Exp Pathol 8: 9506-9511, 2015
14. Deng Z, Hasegawa M, Yamashita Y, Matayoshi S, Kiyuna A, Agena S, Uehara T, Maeda H and Suzuki M: Prognostic value of human papillomavirus and squamous cell carcinoma antigen in head and neck squamous cell carcinoma. Cancer Sci 103 : 2127-2134, 2012.

15. Zheng X, Xing S, Liu XM, Liu W, Liu D, Chi PD, Chen H, Dai SQ, Zhong Q, Zeng MS, et al: Establishment of using serum YKL-40 and SCCA in combination for the diagnosis of patients with esophageal squamous cell carcinoma. BMC Cancer 14: 490, 2014.

16. Guarino M, Di Costanzo GG, Gallotta A, Tortora R, Paneghetti L, Auriemma F, Tuccillo C, Fassina G, Caporaso N and Morisco F: Circulating SCCA-IgM complex is a useful biomarker to predict the outcome of therapy in hepatocellular carcinoma patients. Scand J Clin Lab Invest 77: 448-453, 2017.

17. Chen X, Wang X, He H, Liu Z, Hu JF and Li W: Combination of circulating tumor cells with serum carcinoembryonic antigen enhances clinical prediction of non-small cell lung cancer. PLoS One 10: e0126276, 2015.

18. Brioschi PA, Bischof P, Delafosse C and Krauer F: Squamous-cell carcinoma antigen (SCC-A) values related to clinical outcome of pre-invasive and invasive cervical carcinoma. Int J Cancer 47: 376-379, 1991.

19. Cao L, Wang X, Li S, Zhi Q, Wang Y, Wang L, Li K and Jiang R: PD-L1 is a prognostic biomarker in resected NSCLC patients with moderate/high smoking history and elevated serum SCCA level. J Cancer 8: 3251-3260, 2017.

20. Downward J: Targeting RAS signalling pathways in cancer therapy. Nature Rev Cancer 3: 11-22, 2003.

21. Schmukler E, Kloog Y and Pinkas-Kramarski R: Ras and autophagy in cancer development and therapy. Oncotarget 5: 577-586, 2014.

22. Ye K, Li J, Li X, Chang S and Zhang Z: Ang1/Tie2 induces cell proliferation and migration in human papillary thyroid carcinoma via the PI3K/AKT pathway. Oncol Lett 15: 1313-1318, 2018.

23. Pylayeva-Gupta Y, Grabocka E and Bar-Sagi D: RAS oncogenes: Weaving a tumorigenic web. Nature Rev Cancer 11: 761-774, 2011.

24. Cox AD and Der CJ: Ras history: The saga continues. Small GTPases 1: 2-27, 2010.

25. Frodyma D, Neilsen B, Costanzo-Garvey D, Fisher K and Lewis R: Coordinating ERK signaling via the molecular scaffold Kinase Suppressor of Ras. F1000Res 6: 1621, 2017.

26. Eser S, Schnieke A, Schneider G and Saur D: Oncogenic KRAS signalling in pancreatic cancer. Br J Cancer 111: 817-822, 2014.

27. Margonis GA, Kim Y, Sasaki K, Samaha M, Amini N and Pawlik TM: Codon 13 KRAS mutation predicts patterns of recurrence in patients undergoing hepatectomy for colorectal liver metastases. Cancer 122: 2698-2707, 2016.

28. Christensen TD, Palshof JA, Larsen FO, Poulsen TS, Høgdall E, Pfeiffer P, Jensen BV, Yilmaz MK and Nielsen D: Associations between primary tumor RAS, BRAF and PIK3CA mutation status and metastatic site in patients with chemo-resistant metastatic colorectal cancer. Acta Oncol 57: 1057-1062, 2018.

29. Zaballos MA and Santisteban P: Key signaling pathways in thyroid cancer. J Endocrinol 235: R43-R61, 2017.

30. Liang J, Cai W, Feng D, Teng H, Mao F, Jiang Y, Hu S, Li X, Zhang Y, Liu B and Sun ZS: Genetic landscape of papillary thyroid carcinoma in the Chinese population. J Pathol 244: 215-226, 2018.

31. Sanclemente M, Francoz S, Esteban-Burgos L, Bousquet-Mur E, Djurec M, Lopez-Casas PP, Hidalgo M, Guerra C, Drosten M, Musteanu M, et al: c-RAF ablation induces regression of advanced Kras/Trp53 mutant lung adenocarcinomas by a mechanism independent of MAPK signaling. Cancer cell 33: 217-228, 2018.

32. Xing M: Genetic alterations in the phosphatidylinositol-3 kinase/Akt pathway in thyroid cancer. Thyroid 20: 697-706, 2010.

33. Ning L, Rao W, Yu Y, Liu X, Pan Y, Ma Y, Liu R, Zhang S, Sun H and Yu Q: Association between the KRAS gene polymorphisms and papillary thyroid carcinoma in a Chinese Han population. J Cancer 7: 2420-2426, 2016.

34. Catanzaro JM, Sheshadri N, Pan JA, Sun Y, Shi C, Li J, Powers RS, Crawford HC and Zong WX: Oncogenic Ras induces inflammatory cytokine production by upregulating the squamous cell carcinoma antigens SerpinB3/B4. Nat Commun 5: 3729, 2014.

35. Luo D, Chen H, Li X, Lu P, Long M, Peng X, Lin S, Tan L, Zhu Y, Ouyang N, et al: Activation of the ROCK1/MMP-9 pathway is associated with the invasion and poor prognosis in papillary thyroid carcinoma. Int J Oncol 51: 1209-1218, 2017. 
36. Livak KJ and Schmittgen TD: Analysis of relative gene expression data using real-time quantitative PCR and the $2^{-\Delta \Delta C_{\mathrm{T}}}$ method. Methods 25: 402-408, 2001.

37. Haugen BR, Alexander EK, Bible KC, Doherty GM, Mandel SJ, Nikiforov YE, Pacini F, Randolph GW, Sawka AM, Schlumberger M, et al: 2015 American thyroid association management guidelines for adult patients with thyroid nodules and differentiated thyroid cancer: The american thyroid association guidelines task force on thyroid nodules and differentiated thyroid cancer. Thyroid 26: 1-133, 2016.

38. Ming J, Liu Z, Zeng W, Maimaiti Y, Guo Y, Nie X, Chen C, Zhao X, Shi L, Liu C, et al: Association between BRAF and RAS mutations, and $R E T$ rearrangements and the clinical features of papillary thyroid cancer. Int J Clin Exp Pathol 8: 15155-15162, 2015.

39. La Vecchia C, Malvezzi M, Bosetti C, Garavello W, Bertuccio P Levi $F$ and Negri E: Thyroid cancer mortality and incidence: A global overview. Int J Cancer 136: 2187-2195, 2015.

40. Han X, Wen H, Ju X, Chen X, Ke G, Zhou Y, Li J, Xia L, Tang J, Liang S, et al: Predictive factors of para-aortic lymph nodes metastasis in cervical cancer patients: A retrospective analysis based on 723 para-aortic lymphadenectomy cases. Oncotarget 8: 51840-51847, 2017.

41. Markovina S, Wang S, Henke LE, Luke CJ, Pak SC, DeWees T, Pfeifer JD, Schwarz JK, Liu W, Chen S, et al: Serum squamous cell carcinoma antigen as an early indicator of response during therapy of cervical cancer. Br J Cancer 118: 72-78, 2018.

42. Fassan M, Realdon S, Vianello L, Quarta S, Ruol A, Castoro C, Scarpa M, Zaninotto G, Guzzardo V, Chiarion Sileni V, et al: Squamous cell carcinoma antigen (SCCA) is up-regulated during Barrett's carcinogenesis and predicts esophageal adenocarcinoma resistance to neoadjuvant chemotherapy. Oncotarget 8: 24372-24379, 2017.
43. Sheshadri N, Catanzaro JM, Bott AJ, Sun Y, Ullman E, Chen EI, Pan JA, Wu S, Crawford HC, Zhang J, et al: SCCA1/SERPINB3 promotes oncogenesis and epithelial-mesenchymal transition via the unfolded protein response and IL6 signaling. Cancer Res 74: 6318-6329, 2014

44. Huang L, Zheng M, Liu JH, Xiong Y, Ding H, Tang L and Wang HY: Risk factors and prognosis of IB-IIB cervical carcinoma with common iliac lymph node metastasis. Chin J Cancer 29: 431-435, 2010.

45. Aoki Y, Sasaki M, Watanabe M, Sato T, Tsuneki I, Aida H and Tanaka K: High-risk group in node-positive patients with stage IB, IIA, and IIB cervical carcinoma after radical hysterectomy and postoperative pelvic irradiation. Gynecol Oncol 77: 305-309, 2000

46. Yoon SM, Shin KH, Kim JY, Seo SS, Park SY, Moon SH and Cho KH: Use of serum squamous cell carcinoma antigen for follow-up monitoring of cervical cancer patients who were treated by concurrent chemoradiotherapy. Radiat Oncol 5: 78, 2010.

47. Tripathi $\mathrm{K}$ and Garg M: Mechanistic regulation of epithelial-to-mesenchymal transition through RAS signaling pathway and therapeutic implications in human cancer. J Cell Commun Signal 12: 513-527, 2018.

48. Jeong SH, Hong HS, Kwak JJ and Lee EH: Analysis of RAS mutation and PAX8/PPARg rearrangements in follicular-derived thyroid neoplasms in a Korean population: Frequency and ultrasound findings. J Endocrinol Invest 38: 849-857, 2015. 